\title{
PHENIX W to mu measurements in polarized proton-proton collisions
}

\author{
Ralf SEIDL*† \\ RIKEN \\ E-mail: rseidleriken.jp
}

The PHENIX experiment at RHIC has been taking longitudinally polarized proton-proton collision data at center-or-mass energies of around $\sqrt{s}=500 \mathrm{GeV}$ in the years 2011 to 2013 . One of the main goals of this data-taking period is the access to the sea quark polarization in the nucleon via the parity violating production of real $\mathrm{W}$ bosons. Two decay channels are accessible at PHENIX, at central rapidities $\mathrm{W}$ to electron decays are detected, while at forward rapidities, $1.2<\eta<2.5$ decay muons are detected. These proceedings concentrate on the measurements in the muon arms.

XXII. International Workshop on Deep-Inelastic Scattering and Related Subjects 28 April - 2 May 2014

Warsaw, Poland

\footnotetext{
* Speaker.

${ }^{\dagger}$ for the PHENIX collaboration
} 


\section{Introduction}

The study of the spin structure of the nucleon is one of the main avenues to research the strong interaction in one of the fundamental bound states of QCD and the visible matter in the universe. It has helped us understand that the simple quark model is not sufficient to describe the helicity of the nucleon as quarks' helicities only contribute about $30 \%$ to the total spin of the nucleon. The remainder is expected to be found in gluon spins and orbital angular momentum of quarks and gluons. However, even the sea-quark helicities are not yet too well determined already at intermediate Bjorken $\mathrm{x}$. The reason is that sea quark helicities are mostly accessed in semi-inclusive DIS where the knowledge of the fragmentation functions is required to extract them. Another way to access them is via the party violating real $\mathrm{W}$ production in polarized protonproton collisions without the need of fragmentation functions and at very high scale [1]. The weak interaction only couples to left-handed quarks and right-handed antiquarks and therefore naturally selects (anti)quarks with helicity either parallel or anti-parallel to the nucleons helicity depending on the polarized nucleon's spin direction. Furthermore, neglecting second generation sea quarks in the nucleon, the charge of the produced $\mathrm{W}$ also selects the appropriate flavors such as $u+\bar{d} \rightarrow W^{+}$. The asymmetries for Ws can at leading order be written as:

$$
A_{L}^{W^{+}}=\frac{1}{\langle P\rangle} \frac{N^{+}-N^{-}}{N^{+}+N^{-}} \propto \frac{\Delta \bar{d}\left(x_{1}\right) u\left(x_{2}\right)-\Delta u\left(x_{1}\right) \bar{d}\left(x_{2}\right)}{\bar{d}\left(x_{1}\right) u\left(x_{2}\right)+u\left(x_{1}\right) \bar{d}\left(x_{2}\right)},
$$

where $N^{ \pm}$are the $\mathrm{W}$ count rates for polarized protons with positive(negative) helicity, $\langle P\rangle$ is the average beam polarization and $(\Delta) q(x)$ are the unpolarized and helicity parton distribution functions at momentum fractions $x$ of either the polarized or unpolarized proton. Similarly the $W^{-}$ asymmetry becomes:

$$
A_{L}^{W^{-}}=\frac{1}{\langle P\rangle} \frac{N^{+}-N^{-}}{N^{+}+N^{-}} \propto \frac{\Delta \bar{u}\left(x_{1}\right) d\left(x_{2}\right)-\Delta d\left(x_{1}\right) \bar{u}\left(x_{2}\right)}{\bar{u}\left(x_{1}\right) d\left(x_{2}\right)+d\left(x_{1}\right) \bar{u}\left(x_{2}\right)} .
$$

If one had the opportunity to directly measure the Ws one could even further separate the asymmetries into forward going (related to the polarized nucleon direction) being due to the predominantly valence quark helicities and backward going mostly due to sea quark helicities. However, this requires a near $4 \pi$ acceptance detector to identify the Ws via the missing transverse momentum carried away by the neutrinos and is not available in the current PHENIX detector.

\section{PHENIX W measurements}

The PHENIX experiment consists of two central detectors $|\eta|<0.35$ of $\pi / 2$ azimuthal coverage and two muon arms of about $1.2<|\eta|<2.5$. The muon arms contain a forward vertex detector (FVTX) since 2012, about $1.1 \mathrm{~m}$ Iron and Steel absorber followed by three layers of muon trackers (MuTR) within a radial magnetic field and a muon identifier (MuID) of 5 layers of Iarocci tubes sandwiched with Iron plates. Before the muon magnet and behind the last identifier station two planes of resistive plate counters (RPC) are used to ensure the correct timing relevant for spin measurements and to work as part of the trigger system together with a fast readout of the muon tracker. With this acceptance only W decay electrons (central) and muons (forward/backward) can 
be reconstructed and the simple distinction of forward going Ws corresponding to valence quarks and backward going Ws to sea quarks gets substantially smeared due to the $\mathrm{W}$ decay kinematics again governed by the parity violation especially for positive Ws. The corresponding formulas become:

$$
A_{L}^{W^{+} \rightarrow \mu^{+}} \propto \frac{(1+\cos \theta) \Delta \bar{d}\left(x_{1}\right) u\left(x_{2}\right)-(1-\cos \theta) \Delta u\left(x_{1}\right) \bar{d}\left(x_{2}\right)}{(1+\cos \theta) \bar{d}\left(x_{1}\right) u\left(x_{2}\right)+(1-\cos \theta) u\left(x_{1}\right) \bar{d}\left(x_{2}\right)}
$$

and

$$
A_{L}^{W^{-}} \propto \frac{(1-\cos \theta) \Delta \bar{u}\left(x_{1}\right) d\left(x_{2}\right)-(1+\cos \theta) \Delta d\left(x_{1}\right) \bar{u}\left(x_{2}\right)}{(1-\cos \theta) \bar{u}\left(x_{1}\right) d\left(x_{2}\right)+(1+\cos \theta) d\left(x_{1}\right) \bar{u}\left(x_{2}\right)}
$$

The decay distributions are visualized in Fig. 1 where one sees the rather different rapiditytransverse momentum distributions for positive and negative decay muons. One also sees in this

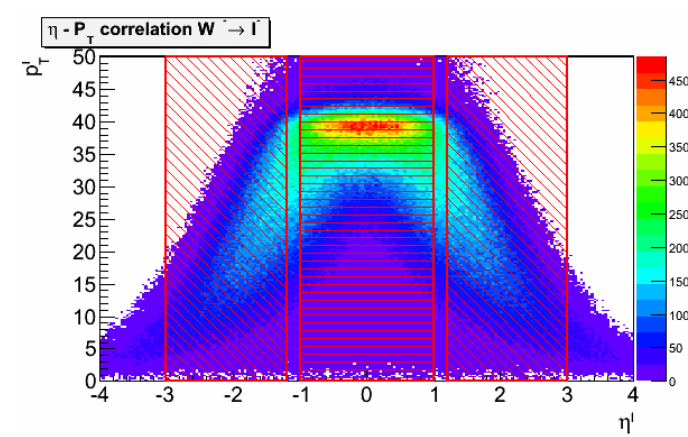

$P_{\mathrm{T}}$ projection $-1.0<\eta<1.0$

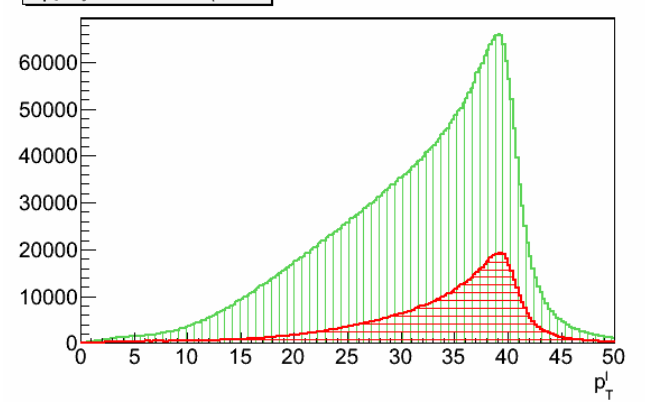

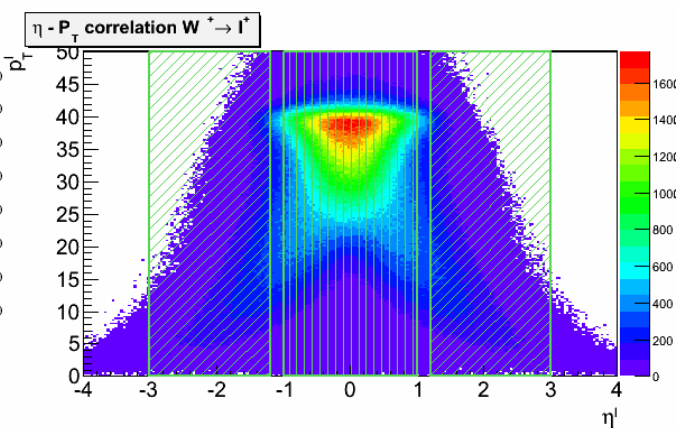

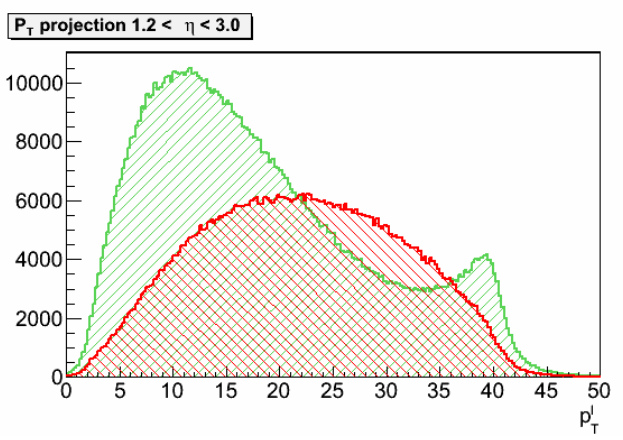

Figure 1: Top panel: 2-dimensional rapidity-transverse momentum distirbutions for $W^{-} \rightarrow \mu^{-}$decays (left) and $W^{+} \rightarrow \mu^{+}$decays (right) from Pythia 6.4. Bottom panel: lepton transverse momentum projections for central rapidities $(|\eta|<1$, left) and forward rapidities $(1.2<|\eta|<3$, right). The positive decay muons are displayed in the green shaded regions, while the negative decay muons are displayed in the green shaded regions.

figure, that for the central region one observes a clean Jacobian peak at half the $\mathrm{W}$ mass, while in the forward region only a hint of a Jacobian is still visible in the positive decay leptons. Including detector smearing one therefore does not have the luxury to search for a Jacobian peak to identify the $\mathrm{W}$ decay muons. Consequently the analysis of the forward $\mathrm{W}$ decay muons mostly concentrates on reducing and understanding backgrounds. There are two types of backgrounds. The first are real muon backgrounds created by mostly heavy flavor decays, onium decays as well as DrellYan/Z boson production. With the exception $\mathrm{Z}$ decay muons which cannot be distinguished all other decay muons have transverse momentum distributions which fall off more rapidly than $\mathrm{W}$ 
decay muons which are then dominating at transverse momenta of about 15 to $20 \mathrm{GeV}$. However, due to the moderate momentum resolution a substantial background due to the real muons from non $\mathrm{W}$ processes remains.

The second type of backgrounds is related to lower energetic hadrons which occasionally survive all absorbing material in front of the muon tracker and decay within the tracking volume mimicking a nearly straight and therefore high momentum track. While the substantial amount of absorbing material before and after the muon tracker removes the majority of those events, the hadronic cross sections for low momentum hadrons are so much higher than the $\mathrm{W}$ production cross section. Therefore this becomes the dominating background in this analysis and before applying tighter selection criteria has muon candidate yields of up to three orders of magnitude higher than the expected signal. The effort to reduce this contribution mostly concentrates on the amount of multiple scattering in the absorbing materials. The most important quantities are the matching of the projected track to the initial vertex, the matching between muon tracker track and muon identifier "road" as well as the matching to clusters in the most downstream RPC station. In addition to these values various other quantities related to the general track quality and the FVTX if in its acceptance are used together to form an initial likelihood ratio between signal Monte Carlo and data before cuts which is dominated by background. For the further analysis muon candidates with a likelihood ratios of above 0.92 are selected which is about $90 \%$ efficient for $\mathrm{W}$ decay signal muons. Afterwards an un-binned maximum likelihood fit is performed in rapidity and modified sagitta using template distributions from $\mathrm{MC}$ for the $\mathrm{W}+\mathrm{Z}$ signal, hadronic background distributions extrapolated from data at lower likelihoods and fixed other muon backgrounds as obtained from MC confirmed with di-muon data. Signal to background ratios are about $1 / 3$ and slightly higher when removing the modified sagitta sidebands still used in the fit. The asymmetries are then calculated on this sample and the dilution due to background is corrected for after testing that indeed no parity violating asymmetry is present in the background.

The preliminary asymmetries for the 2012 running period, consisting of about $42 \mathrm{pb}^{-1}$ without collision vertex restriction are displayed in Fig. 2. The analysis of the 2013 data with about 5 times as much statistics is currently ongoing.

\section{Outlook}

When the full PHENIX polarized proton proton $500 \mathrm{GeV}$ dataset will be analyzed it is expected, that the uncertainties on the sea quark polarizations will be further reduced. The expected improvements can be seen in [9] after including the published $W \rightarrow e$ data from PHENIX [2] and STAR [3, 4] as well as in [11] when including the recently submitted STAR 2012 data [10].

\section{References}

[1] C. Bourrely and J. Soffer, Phys. Lett. B 314 (1993) 132.

[2] A. Adare et al. [PHENIX Collaboration], Phys. Rev. Lett. 106 (2011) 062001

[3] M. M. Aggarwal et al. [STAR Collaboration], Phys. Rev. Lett. 106 (2011) 062002

[4] L. Adamczyk et al. [STAR Collaboration], Phys. Rev. D 85 (2012) 092010 

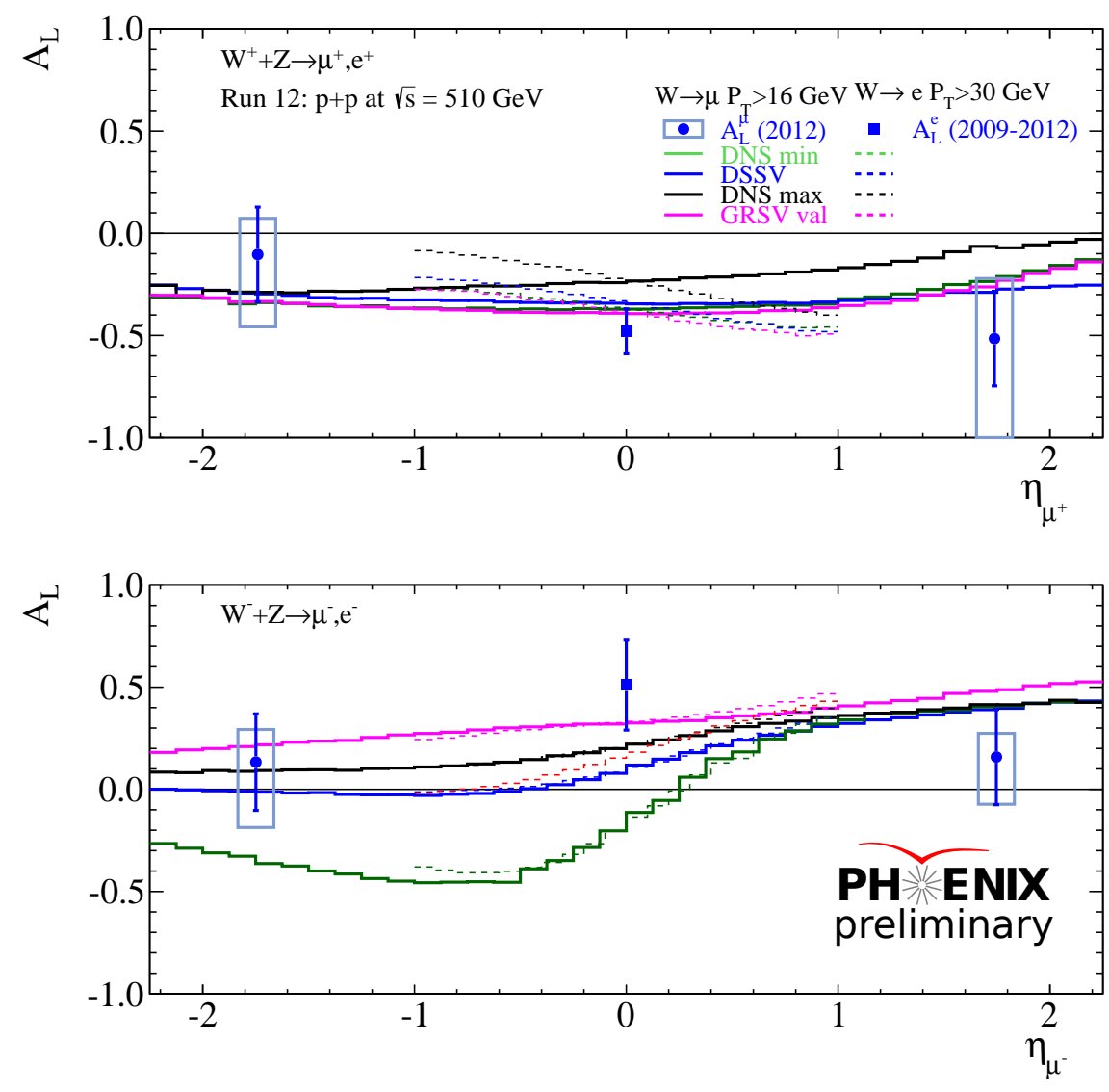

Figure 2: Preliminary forward $W \rightarrow \mu$ from the 2012 data taking period and preliminary $W \rightarrow e$ asymmetries from the 2009- 2012 data taking periods as a function of the rapidity. The various lines correspond to parametrizations from global helicity analysis DSSV [5](blue), DNS[6] (darkgreen and black for two types of fragmentation function parametrizations) and GRSV[7] (purple) using RHICBOS [8] to calculate the asymmetries. The continuous lines correspond to transverse lepton momenta above $16 \mathrm{GeV}$ while the dashed lines correspond to lepton momenta above $30 \mathrm{GeV}$ as applied in the PHENIX forward and central W analyses respectively.

[5] D. de Florian, R. Sassot, M. Stratmann and W. Vogelsang, Phys. Rev. D 80 (2009) 034030

[6] D. de Florian, G. A. Navarro and R. Sassot, Phys. Rev. D 71 (2005) 094018

[7] M. Gluck, E. Reya, M. Stratmann and W. Vogelsang, Phys. Rev. D 53 (1996) 4775

[8] P. M. Nadolsky and C. P. Yuan, Nucl. Phys. B 666 (2003) 31

[9] E. C. Aschenauer, A. Bazilevsky, K. Boyle, K. O. Eyser, R. Fatemi, C. Gagliardi, M. Grosse-Perdekamp and J. Lajoie et al., arXiv:1304.0079 [nucl-ex].

[10] L. Adamczyk et al. [STAR Collaboration], arXiv:1404.6880 [nucl-ex].

[11] E. R. Nocera et al. [The NNPDF Collaboration], arXiv:1406.5539 [hep-ph]. 